\title{
Preise und Auszeichnungen
}

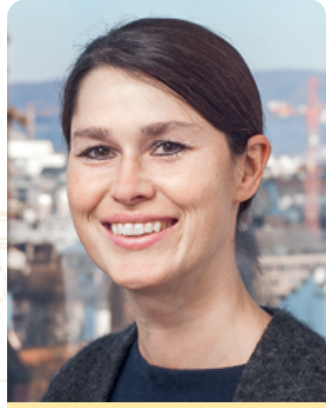

Prof. Mirjam Christ-Crain (Foto: Universitätsspital Basel)

\section{European Journal of Endocrinology Award}

Professorin Mirjam Christ-Crain, die stellvertretende Leiterin der Klinik für Endokrinologie, Diabetologie und Metabolismus und Co-Leiterin des Departements Klinische Forschung des Universitätsspitals Basel, wurde für ihre Forschung zum Vasopressinhaushalt ausgezeichnet. Sie erhielt einen der wichtigsten Preise für Endokrinologie in Europa, den «European Journal of Endocrinology Award».

Die Forschungsarbeiten wurden an mehreren Zentren in Europa und Südamerika durchgeführt und bereits im New England Journal of Medicine und dem Lancet veröffentlicht.

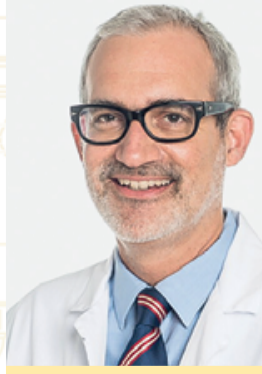

Prof. Marius Keel (Foto: Klinik Hirslanden) Bone \& Joint Surgery und JBJS Essential Surgical Techniques für den besten technischen Artikel mit dem «JBJS Essential Surgical Techniques Editor's Choice Technique Award» ausgezeichnet. Der Artikel erschien unter dem Titel «The Pararectus Approach - A New Concept» (Der PararectusZugang - ein neues Konzept) und befasst sich mit dem minimalinvasiven von Prof. Keel entwickelten Pararectus-Zugang, der bei Frakturen der Hüftgelenkspfanne eine bessere Darstellung der Fraktur ermöglicht und mit dem auch über 60-jährige Patientinnen und Patienten erfolgreich behandelt werden können.
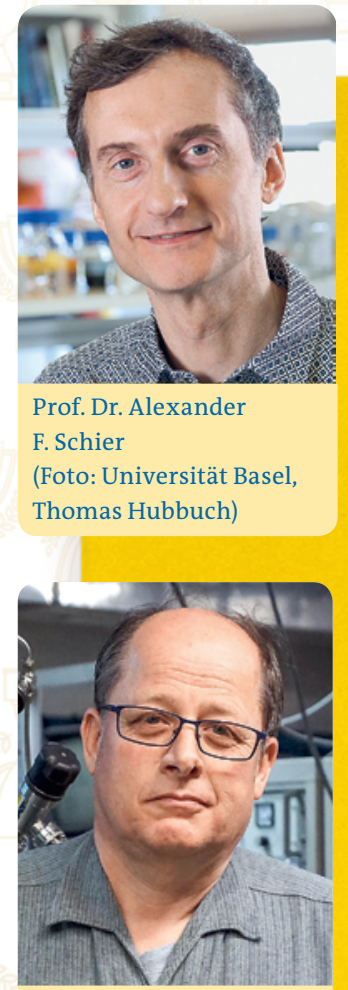

Prof. Dr. Ernst Meyer (Foto: Klinik Hirslanden)

\section{ERC Advanced Grant}

Der Europäische Forschungsrat fördert zwei Wissenschaftler der Universität Basel Prof. Alex Schier und Prof. Ernst Meyer - mit je einem hoch dotierten ERC Advanced Grant. Alex Schier ist Professor für Zell- und Entwicklungsbiologie am Biozentrum der Universität Basel und dessen Direktor; zugleich leitet er eine Forschungsgruppe an der Harvard University in Cambridge (USA). Sein ERC-Projekt ist dem Entwicklungsverlauf einzelner Zellen gewidmet und zielt darauf ab, die Logik der Zelldifferenzierung zu entschlüsseln.

Mit Hilfe der neuen Analysemethoden möchten die Forscher nun einen umfassenden Einblick gewinnen, wie die zelluläre Vielfalt entsteht und wie sich spezialisierte Zellen mit einer genau definierten Aufgabe entwickeln. Dazu analysieren sie mit genetischen Verfahren die Stammbäume und Entwicklungswege sowie ihre Wechselbeziehung.

Am Modell von Zebrafischen durchgeführt, soll das Vorhaben dazu beitragen, eine umfassende Gesamtsicht der Zellentwicklung bei Wirbeltieren zu ermöglichen.

Rasterkraftmikroskope bestehen aus einer Spitze, die in einem Abstand von wenigen Nanometern über die Oberfläche einer Probe geführt wird. Anhand der atomaren Kräfte, die auf die Spitze wirken und den Sensor verbiegen, lassen sich atomare Details abbilden und Nanostrukturen manipulieren.

Ernst Meyer ist Professor für Experimentalphysik an der Universität Basel und Mitglied der Geschäftsleitung des Swiss Nanoscience Institute (SNI). Im Rahmen seines ERCProjekts möchte er die Technik so weiterentwickeln, dass sich damit die Mechanismen der Energiedissipation - der Umwandlung von Energie in Wärme - beobachten und aufklären lassen. Im Fokus stehen Reibungseffekte in mikroskopischen Dimensionen, insbesondere Energieverluste, die zwischen der Oberfläche und der Spitze des Rasterkraftmikroskops entstehen. 